\title{
Postoperative pain is undertreated: results from a local survey at Jordan University Hospital
}

I.M. Massad, ${ }^{7}$ T.M. Mahafza, ${ }^{2}$ S.A. Abu-Halawah, ${ }^{1}$ B.A. Attyyat, ${ }^{7}$ S.M. Al-Ghanem, ${ }^{1}$ M.M. Almostafa ${ }^{1}$ and A. S Al-Oweidi ${ }^{1}$

$$
\begin{aligned}
& \text { النقص في معالجة الألم التالي للجراحة: نتائج مستمدة من مسح محلي في مستشفى الجامعة الأردنية }
\end{aligned}
$$

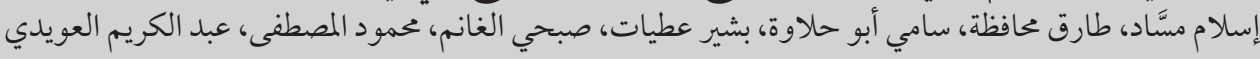

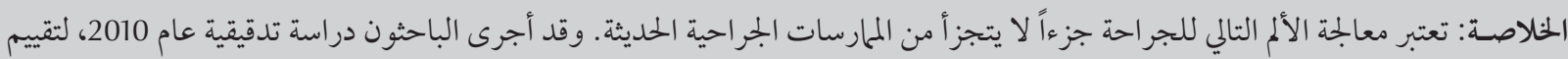

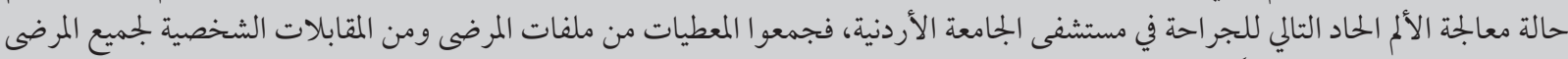

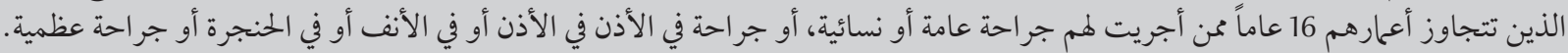

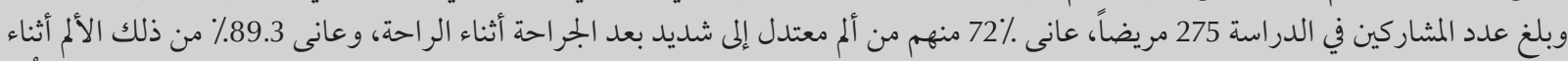

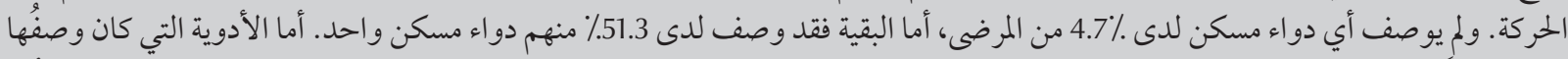

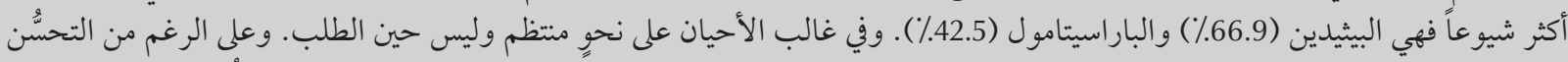

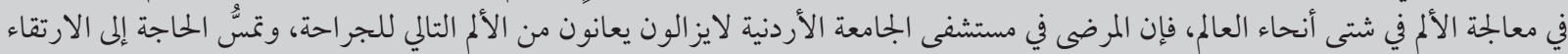

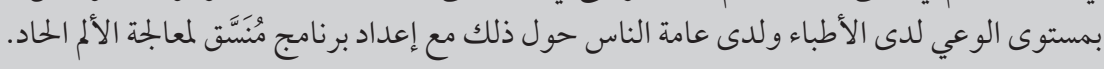

ABSTRACT Postoperative pain management is nowadays considered an integral part of modern surgical practice. An audit was made in 2010 to assess the status of acute postoperative pain management at Jordan University Hospital. Data were collected from patients' files and through face-to-face interviews of all patients aged over 16 years who underwent general, gynaecological, ear-nose-throat and orthopaedic surgery. Of 275 patients, $72.0 \%$ experienced moderate to severe pain postoperatively at rest and $89.3 \%$ on movement. No analgesics were prescribed to $4.7 \%$ of the patients and of the remainder, a single analgesic was prescribed to $51.5 \%$. Pethidine and paracetamol were the drugs most commonly prescribed (to $66.9 \%$ and $42.5 \%$ of patients respectively), most often on a regular schedule rather than on-demand. Despite improvements in pain management worldwide, patients at this hospital were still suffering from postoperative pain. Awareness among professionals and the public is needed and a structured acute pain management programme is essential.

Traitement insuffisant de la douleur postopératoire : résultats d'une enquête locale à I'Hôpital universitaire de Jordanie

RÉSUMÉ La prise en charge de la douleur postopératoire est aujourd'hui considérée comme faisant partie intégrante de la pratique chirurgicale moderne. Un audit a été conduit en 2010 pour évaluer la prise en charge de la douleur postopératoire aiguë à l'Hôpital universitaire de Jordanie. Des données ont été recueillies à partir des dossiers médicaux des patients et au cours d'entretiens individuels avec tous les patients de plus de 16 ans ayant subi une intervention chirurgicale générale, gynécologique, orthopédique ou de la sphère ORL. Sur 275 patients, $72,0 \%$ ont ressenti une douleur postopératoire d'intensité modérée à sévère au repos et 89,3\% en action. Aucun analgésique n'a été prescrit à 4,7 \% des patients. Pour les patients ayant reçu un traitement antidouleur, un simple analgésique a été prescrit à 51,5\% d'entre eux. La péthidine et le paracétamol étaient les médicaments les plus fréquemment prescrits (à 66,9\% et 42,5\% des patients respectivement), le plus souvent à un horaire régulier plutôt qu'à la demande. Malgré des améliorations de la prise en charge de la douleur au niveau mondial, les patients admis dans cet hôpital souffraient encore de douleur postopératoire. Une sensibilisation des professionnels et de la population est requise et un programme structuré de prise en charge de la douleur aiguë est essentiel. 


\section{Introduction}

Pain is a personal, subjective experience that involves sensory, emotional and behavioural factors associated with actual or potential tissue injury [1]. In 2004 during the first Global Day Against Pain the World Health Organization, along with the International Association for the Study of Pain (IASP) and the European Federation of IASP Chapters, issued a joint declaration that "the relief of pain should be a human right" [2]. Postoperative pain management is nowadays considered to be an integral part of modern surgical practice. Unrelieved postoperative pain has been found to have profound implications, which includes clinical and psychological changes that increase morbidity and mortality and hence the costs of care and decrease the quality of life [3].

Adequate institutional attention to pain assessment and treatment has become a requirement of hospital accreditation [4] and many guidelines have been issued in different regions of the world $[5,6]$. These guidelines promote standardization of procedures, with recommendations for proactive planning such as carrying out surveys about the effectiveness of postoperative pain management, holding pain management training courses [5] and using effective methods for pain relief such as pre-emptive analgesia [7], multimodal analgesia [8], patient-controlled analgesia $[9]$ or others.

Jordan University Hospital was accredited by the Joint Commission on Accreditation of Healthcare Organizations (JCAHO) in 2010. This involved a considerable amount of work on acute pain management including that for the postoperative period. Nevertheless, an understanding of the postoperative pain experience from a patient's perspective is important if health care professionals are to identify ways of improving care. The objective of the current survey was to evaluate the status of postoperative pain relief and to assess the types and routes of drugs administered for this purpose at Jordan University Hospital.

\section{Methods}

\section{Study design and setting}

The survey was conducted at Jordan University Hospital in February 2010 following approval for the study by the research committee of the Faculty of Medicine at the University of Jordan, This observational, non-interventional, longitudinal, single-centre survey involved patients who were more than 16 years old undergoing major or minor general, gynaecological, ear-nose-throat (ENT) or orthopaedic surgery.

\section{Sample}

For the purpose of this survey, we enrolled 275 adult patients. This sample size was sufficient to provide a high precision for estimating the proportion of patients with pain at rest or on movement with a margin of error not exceeding $5 \%$, assuming a level of confidence of $95 \%$ and an expected proportion of patients with pain of $70 \%$. The survey excluded patients who were admitted to the intensive care unit, those who were re-operated within the same day of surgery or those whose charts were unavailable or missing. None of the patients had patient-controlled analgesia, which was not available at the hospital at the time of the study.

\section{Data collection}

Data were collected from the patients' charts about: demographic variables, surgery carried out (surgical procedure, date of surgery and type of anaesthesia used) and postoperative analgesia prescribed. The data about analgesia included: what analgesics were prescribed, the route and mode of administration (systematically in a scheduled fashion or on-demand by the patient), the number of prescribed analgesics, total daily dose of analgesics (mg per day), any changes in the analgesic treatment prescribed and the reason for change.

Data related to pain assessment was collected through face-to-face interviews on the ward during the day of the survey. For those patients who spent the night at the hospital this was done 4 and 8 hours after the patient was transferred from the recovery room and in the morning of the first postoperative day. Patients who were discharged home on the same day of surgery had a single face-to-face interview before hospital discharge. Assessment of pain severity was done during the interview using one of the following pain scales (evaluators chose which scale to use in each case):

- Verbal rating scale. The patient was asked to choose from verbal descriptions of pain: "no pain", "mild pain", "moderate pain" and "severe pain" [10].

- Numerical rating scale. The patient was asked to choose a number from $0-10$ that best reflected the level of pain felt, using a scale labelled from "no pain" (score 0) to "worst pain possible" (score 10). For the analysis, scores between $0-3$ were considered as "mild pain", 4-7 as "moderate pain" and $8-10$ as "severe pain" [10].

- Visual analogue scale. This uses a vertical or horizontal line with words that convey "no pain" at one end and "worst pain" at the opposite end. The patient is asked to place a mark along the line that indicates the level of pain experienced.

Adverse events considered to be related to analgesics and requiring symptomatic treatment, e.g. nausea, vomiting, urinary retention, respiratory depression, ileus, itching, sedation or sleepiness were also collected at the time of the interview.

The interviews and chart reviews were done by 3 senior anaesthesia residents in their 4th year of training, who were involved neither in conducting anaesthesia for the patients nor in 
prescribing postoperative analgesia for them. These residents were trained by the researchers to interview the patients and to review the patients' charts, then to fill the data on a preset software form prepared by the PATHOS-SIMPATHI European Steering Committee [11]. The SIMPATHI programme consists of a programme toolkit that includes materials covering personnel training, patient information, protocol development and pain evaluation follow-up areas. This programme was based on the need of improvement identified in a previous postoperative analgesic therapy observational survey (PATHOS), which was conducted to assess postoperative pain management practices on surgical needs in Europe, and to identify areas requiring improvement in health care institutes.

\section{Results}

A total of 275 adult patients were eligible for the study. The mean age was 40.5 (SD 15.8) years. Most patients who participated were women (66.5\%). General anaesthesia was conducted on $92.4 \%$ of the patients while only $7.6 \%$ of them had local or regional blocks for the included types of surgeries (Table 1).

A total of 424 prescriptions for analgesics were given to 262 patients. No analgesics were prescribed to $13 / 275$ patients (4.7\%). Of those patients who received pain medication, 135/262 (51.5\%) received a single drug for postoperative pain relief and 127 (48.5\%) of them had multiple analgesics. The medication(s) was changed in $17 / 262$ (6.5\%) of the patients, in 9 cases due to lack of safety of the prescribed drug(s), in 7 cases due to lack of efficacy of the drug and in 1 patient the reason was not known.

Experience of pain was evaluated by the verbal rating scale in $244 / 275$ patients $(88.7 \%)$, while the numeric rating scale was used in 31 patients

\begin{tabular}{lcc}
\hline Table 1 Background characteristics of the studied patients $(\boldsymbol{n}=\mathbf{2 7 5})$ & \\
\hline Variable & No. of patients & $\%$ \\
Sex & 92 & 33.5 \\
Male & 183 & 66.5 \\
Female & & \\
Type of anaesthesia & 254 & 92.4 \\
General & 21 & 7.6 \\
Regional & & \\
Type of surgery & 24 & 8.7 \\
Major abdominal & 75 & 27.3 \\
Minor abdominal & 22 & 8.0 \\
Major orthopaedic & 35 & 12.7 \\
Minor orthopaedic & 39 & 14.2 \\
Major gynaecological & 29 & 10.5 \\
Minor gynaecological & 3 & 1.1 \\
Major ENT & 48 & 17.5 \\
Minor ENT & & \\
\hline
\end{tabular}

$E N T=$ ear-nose-throat.
(11.3\%) and the visual analogue scale was not used. Pain evaluation assessed at rest and on movement, showed that $72.0 \%$ of the patients suffered from moderate to severe pain at rest, and $88.7 \%$ suffered moderate to severe pain on movement. It was not feasible to assess pain on movement in 4 patients (Table 2).

Pethidine was the most commonly prescribed drug, prescribed to $184 / 275$ patients (66.9\%). It was given on a regular schedule in $151 / 184$ patients (82.1\%) and on request from the patient in 33 cases (17.9\%). Pethidine was always given intramuscularly (Table 3 ). Another commonly prescribed drug was paracetamol, which was given to $117 / 275$ patients ( $42.5 \%$ ). This medication was given systematically in 93/117 patients $(80.3 \%)$, through the oral, intravenous and rectal routes in 100 , 15 and 2 patients respectively (Table 3). Other medications prescribed their daily doses and route of administration, and whether given systematically or ondemand, are shown on Table 3.

The survey also showed that $149 / 275$ patients $(54.2 \%)$ had at least 1 analgesic-related side-effect. Of those nausea and vomiting together ranked first; occurring in 76/149 patients (51.0\%). Other analgesia related sideeffects are shown in Table 4.

\begin{tabular}{lcc}
\hline Table 2 Intensity of postoperative pain among the studied patients $(\boldsymbol{n}=\mathbf{2 7 5})$ \\
\hline Pain intensity & No. of patients & $\%$ \\
At rest & 53 & 19.3 \\
$\quad$ Severe pain & 145 & 52.7 \\
Moderate pain & 77 & 28.0 \\
Mild or no pain & & \\
On movement & 80 & 29.1 \\
Severe pain & 164 & 59.6 \\
Moderate pain & 27 & 9.8 \\
Mild or no pain & 4 & 1.5 \\
Could not be assessed &
\end{tabular}




\begin{tabular}{|c|c|c|c|c|c|c|c|c|c|}
\hline \multirow[t]{3}{*}{ Medication } & \multirow{2}{*}{\multicolumn{2}{|c|}{$\begin{array}{l}\text { Total times } \\
\text { prescribed }^{\mathrm{a}}\end{array}$}} & \multicolumn{2}{|c|}{ Delivery scheme } & \multicolumn{4}{|c|}{ Route of administration } & \multirow{3}{*}{$\begin{array}{c}\text { Daily dose (mg) } \\
\text { Mean (SD) }\end{array}$} \\
\hline & & & \multirow{2}{*}{$\begin{array}{c}\text { Systematically } \\
\text { No. }\end{array}$} & \multirow{2}{*}{$\begin{array}{c}\text { On-demand } \\
\text { No. }\end{array}$} & \multirow{2}{*}{$\begin{array}{l}\text { i.m. } \\
\text { No. }\end{array}$} & \multirow{2}{*}{$\begin{array}{l}\text { i.v. } \\
\text { No. }\end{array}$} & \multirow{2}{*}{$\begin{array}{l}\text { Oral } \\
\text { No. }\end{array}$} & \multirow{2}{*}{$\begin{array}{l}\text { Other } \\
\text { No. }\end{array}$} & \\
\hline & No. & $\%$ & & & & & & & \\
\hline Pethidine & 184 & 66.9 & 151 & 33 & 184 & - & - & - & 407 (172) \\
\hline Paracetamol & 117 & 42.5 & 93 & 24 & - & 15 & 100 & $2^{b}$ & 2846 (610) \\
\hline Diclofenac & 74 & 26.9 & 38 & 36 & 71 & - & 3 & - & $152(40)$ \\
\hline Tramadol & 30 & 10.9 & 9 & 21 & 22 & 1 & 7 & - & $132(52)$ \\
\hline Morphine & 11 & 4.0 & 8 & 3 & - & 11 & - & - & $61(43)$ \\
\hline Bupivacaine & 4 & 1.5 & 4 & 0 & - & - & - & $4^{c}$ & $150(0)$ \\
\hline Ibuprofen & 2 & 0.7 & 1 & 1 & - & 2 & - & - & $1200(0)$ \\
\hline Lidocaine & 1 & 0.4 & 1 & 0 & - & - & - & $1^{d}$ & $150(0)$ \\
\hline Celecoxib & 1 & 0.4 & 1 & 0 & - & - & 1 & - & $400(0)$ \\
\hline
\end{tabular}

${ }^{a}$ The table shows number (\%) of patients; some patients received more than 1 analgesic.

${ }^{b}$ Rectal; 'Epidural; ${ }^{d}$ Infiltration.

i.m. $=$ intramuscular; i.v. = intravenou, $S D=$ standard deviation.

\section{Discussion}

As Harmer and Davies demonstrated, the first step in improving acute postoperative pain management is to know the current practice at the institution [12]. This was therefore the aim of the audit conducted here. We found that with the current standard of postoperative care in our hospital, $72.0 \%$ of all patients surveyed experienced moderate to severe postoperative pain at rest. These results were very concerning. Similar international surveys suggested that throughout the world postoperative pain continues to be undermanaged. Jeffery et al. assessed patients' postoperative pain experience and the status of acute pain management, and found that approximately $80 \%$ of the patients experienced acute postoperative pain, of who $86 \%$ had moderate to severe pain [13]. Tsui et al.'s earlier study on 1443 surgical patients showed that $75 \%-100 \%$ of these patients experienced moderate to severe pain, and the authors concluded that pain control was far from ideal [14]. After the implementation of the Pain-Free Hospital Project in Germany in 2003, a study on the quality of pain management in 25 German hospitals between 2004-06 showed that about $30 \%$ of patients who had undergone surgery had moderate to severe pain at rest and $55 \%$ of them experienced pain on movement [15]. It is the responsibility of those health care providers or physicians who are involved in acute pain management to identify patients in pain and initiate simple but effective protocols for different

Table 4 Side-effects of postoperative prescribed analgesics in patients with at least 1 adverse event $(n=149)$

\begin{tabular}{lcc} 
Side-effect & No. of patients & $\%$ \\
Nausea and vomiting & 76 & 51.0 \\
Nausea only & 49 & 40.3 \\
Vomiting only & 3 & 2.0 \\
Ileus & 2 & 1.3 \\
Itching & 2 & 1.3 \\
Urinary retention & 0 & 0.0 \\
Respiratory depression & 0 & 0.0 \\
Sedation/sleepiness & 21 & 14.1 \\
\hline
\end{tabular}

patients, with different presentations and levels of pain tolerance. These protocols were found to be effective in different studies $[5,6,12,16]$.

Our results also showed that pain was managed suboptimally with a limited variety of pain medications; that is, a single medication was used in about half of cases (51.5\%). Pethidine was still used in most of our patients (66.9\%), despite its known side-effects, especially nausea and vomiting [17]. This was evident in our survey results showing that $54.2 \%$ of our patients suffered from side-effects of nausea, vomiting or, more commonly, both. A combination of various analgesic agents and or delivery techniques with different mechanisms of action that enhance analgesia and reduce the side-effect of each drug or technique is needed: the so-called multimodal analgesia [18].

Some of the drawbacks of our survey were that we did not compare the pain control differences between different surgical specialties, and we did not study the patients' trend of pain over time. However, the primary goal of the study was to assess the status of acute postoperative pain control in the 4 specialties studied, and to assess whether any of the surveyed patients had any attack of severe, moderate or mild pain. Another limitation was that the pain 
evaluators used different pain scales for different patients and these scales may vary in sensitivity.

In conclusion, severe postoperative pain was common among Jordan
University Hospital patients. The pain treatment polices at the hospital are clearly inadequate, as we are still depending on traditional, less effective methods for postoperative pain control.
The data showed an urgent need to reassess our policies in postoperative pain management, our treatment options and the medication regimens.

Competing interests: None declared.

\section{References}

1. Merskey H, Ogduk N. Classification of chronic pain. Seattle, International Association for the Study of Pain Press, 1994:210.

2. Brennan F, Carr DB, Cousins M. Pain management: a fundamental human right. Anesthesia and Analgesia. 2007, 105(1):205-221.

3. Costantini $\mathrm{R}$ et al. Controlling pain in the post-operative setting. International Journal of Clinical Pharmacology and Therapeutics, 2011, 49:116-127.

4. Curtiss CP. JCAHO: meeting the standards for pain management. Orthopedic Nursing, 2001, 20:27-30, 41.

5. Savoia G et al.; SIAARTI Study Group. Postoperative pain treatment SIAARTI Recommendations 2010. Short version. Minerva Anestesiologica, 2010, 76:657-667.

6. Guevara-López U et al. Parámetros de práctica para el manejo del dolor agudo perioperatorio. [Practice guidelines for the management of acute perioperative pain]. Cirugia y Cirujanos, 2005, 73:223-232.

7. Ong CK et al. The efficacy of preemptive analgesia for acute postoperative pain management: a meta-analysis. Anesthesia and Analgesia, 2005, 100:757-773.

8. Buvanendran A, Kroin JS. Multimodal analgesia for controlling acute postoperative pain. Current Opinion in Anaesthesiology, 2009, 22:588-593.

9. Momeni M, Crucitti M, De Kock M. Patient-controlled analgesia in the management of postoperative pain. Drugs, 2006, 66:2321-2337.

10. Breivik $\mathrm{H}$ et al. Assessment of pain. British Journal of Anaesthesia, 2008, 101(1):17-24.
11. Benhamou D et al. Postoperative Analgesic THerapy Observational Survey (PATHOS): a practice pattern study in 7 central/ southern European countries. Pain, 2008, 136:134-141.

12. Harmer M, Davies KA. The effect of education, assessment and a standardised prescription on postoperative pain management. The value of clinical audit in the establishment of acute pain services. Anaesthesia, 1998, 53:424-430.

13. Apfelbaum JL et al. Postoperative pain experience: results from a national survey suggest postoperative pain continues to be undermanaged. Anesthesia and Analgesia, 2003, 97:534-540.

14. Tsui SL et al. A clinical audit for postoperative pain control on 1443 surgical patients. Acta Anaesthesiologica Sinica, 1995, 33:137-148

15. Maier $\mathrm{C}$ et al. The quality of pain management in German hospitals. Deutsches Ärzteblatt International, 2010, 107:607-614.

16. Binhas $\mathrm{M}$ et al. Use of a validated reference tool to evaluate postoperative pain management through a quality-improvement program in a university hospital. Journal for Healthcare Quality, 2011, 33:7-13.

17. Latta KS, Ginsberg B, Barkin RL. Meperidine: a critical review. American Journal of Therapeutics, 2002, 9:53-68.

18. Buvanendran A, Kroin JS. Multimodal analgesia for controlling acute postoperative pain. Current Opinion in Anaesthesiology, 2009, 22(5):588-593. 